\title{
An Experimental Model for Advanced Ovarian Cancer
}

\author{
TORU SUGIYAMA, TAKASHI NISHIDA, DAISUKE YOKOTA, KIMIO USHIJIMA, \\ KIYOHISA IMAISHI, NOBUO HIRAKAWA, MASAMICHI NAKANAMI, \\ TAKAAKI ODA AND MICHIAKI YAKUSHIJI
}

\author{
Department of Obstetrics and Gynecology, Kurume University, School of Medicine \\ Kurume, 830 Japan
}

Received for publication December 8, 1989

\begin{abstract}
summary: Intraperitoneally transplanted tumors implanted and began developing with ascites in about $62 \%$ of the female rats within 4 to 6 weeks after transplantation. The tumor used in this study was an adenocarcinoma and which originated from a primary ovarian cancer in rats of the same strain (Wistar). The morphology and biological behavior of the tumor were very similar to the tumor in humans. Moreover, the preliminary results with cisplatin therapy indicate that intraperitoneal cancer corresponding to stage III or IV in the FIGO classification is a promising model for experimental therapeutic studies of common epithelial carcinoma at an advanced stage.

Key words: ovarian cancer - intra-peritoneal transplantation - rat - cisplatin-experimental chemotherapy
\end{abstract}

\section{Introduction}

Ovarian cancer at the time of diagnosis is frequently in an advanced stage, approximately $60 \%$ are in stages III or IV (FIGO, 1985). Thus, improvement in treatment is crucial and there is a great need for an experimental model of advanced ovarian cancers.

Kato et al. (1974) successfully induced ovarian adenocarcinomas in rats by direct application of DMBA to the ovary. The induced adenocarcinoma was similar, morphologically and biologically, to human ovarian cancers (Sugiyama et al. 1981; Nishida et al. 1986). Moreover, use of this tumor for experimental chemotherapy has been reported. A combination of in vivo models for assaying antitumor effects of various chemotherapeutic substances is very useful (Sugiyama et al. 1985, 1987).

The present study was undertaken to use the intraperitoneal ovarian cancer as a model for the study of experimental treatments of advanced cancers.

\section{Materials and Methods}

The primary adenocarcinoma was induced in the ovary of a Wistar rat by local application of 7,12-dimethylbenz (a) anthracene (DMBA) to the ovary. After serial transplantation to the backs of rats for more than 30 generations, small pieces of tumors measuring approximately 10 to $15 \mathrm{~mm}$ were implanted into the peritoneal cavities of 25 newborn rats of the same strain.

Twelve of the 16 tumor bearing rats were sacrificed to examine the extent of the cancer during the 4th, 5th and 6th weeks following transplantation. The remaining four were used for therapeutic 
studies with intraperitoneal cisplatin.

Ascites fluid $(1.0 \mathrm{ml})$ was collected in the 4 th week after transplantation and cytologically examined. A biopsy of the tumors was obtained with an exploratory laparotomy. Then, cisplatin $(5 \mathrm{mg} / \mathrm{kg})$ was administered by intraperitoneal instillation. The implanted tumors were removed after aspiration of the ascites at the 5th week after transplantation (Table 1).

The number of nucleated cells was counted with a hemocytometer. Giemsa and Papanicolaou staining after fixation in Bouin fluid were used to observe the cytological changes.

TABLE 1

\section{Experimental Schedule}

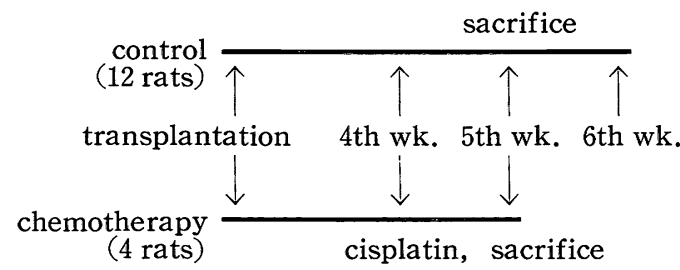

The tissue specimens were fixed in Bouin fluid and stained with hematoxylin and eosin (HE).

\section{Results}

By the 4 th week, intraperitoneal tumors had successfully developed in 16 of 25 rats $(62 \%)$. Two of the four rats sacrificed at the 4th week exhibited intraperitoneal dissemination and bloody ascites (Fig. 1). By the 5th week, the tumor tissue had spread extensively over the peritoneal surface, resembling stage III human ovarian cancer (FIGO). Three tumors examined in the 6th week had progressed beyond the peritoneal cavity. Metastases to the liver and lung were found (Fig. 2a). Metastasis to lymph nodes was observed in 7 of 16 animals. Virchow metastasis was observed in two rats at the 6 th week after transplantation (Table 2 and Fig. 2b).

The cytological specimens showed papillary clusters of neoplastic cells resembling human ovarian adenocarcinoma (Fig. 3).

TABLE 2

Extention of the cancers of 16 tumor-bearing rats

\begin{tabular}{l|c|c|c|c|c|c}
\hline & case & $\begin{array}{c}\text { intraperitoneal } \\
\text { dissemination }\end{array}$ & $\begin{array}{c}\text { malignant* } \\
\text { ascites }\end{array}$ & lymph node & lung & liver \\
\hline \multirow{5}{*}{ 4th week } & 1 & + & + & + & - & - \\
& 2 & + & + & - & - & - \\
& 3 & - & - & - & - & - \\
& 4 & - & - & - & - & - \\
\hline \multirow{5}{*}{ 5th week } & 1 & + & + & + & - & + \\
& 2 & + & + & + & - & - \\
& 3 & + & + & + & - & - \\
\hline & 4 & - & - & - & - & - \\
6 th week & 1 & + & + & + & - & + \\
& 2 & + & + & + & + & - \\
\end{tabular}

$*+$ : ascites $>1.0 \mathrm{ml} * *$ Virchow metastasis $(+)$ 

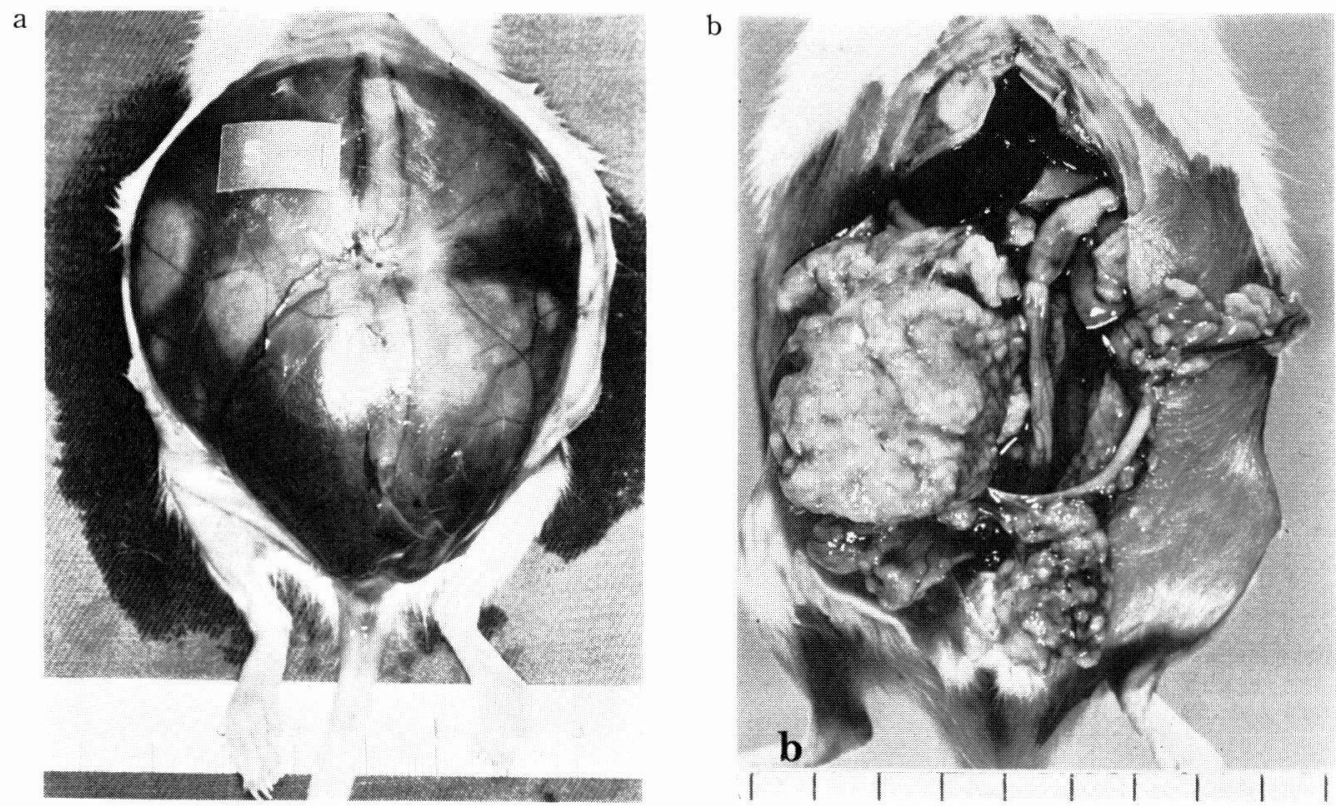

Fig. 1. Rats sacrificed at 4 weeks after transplantation

a) Bloody ascites and intraperitoneal tumors were seen beneath the peritoneum.

b) The transplanted tumor and its dissemination accompanied by malignant ascites were seen.

a

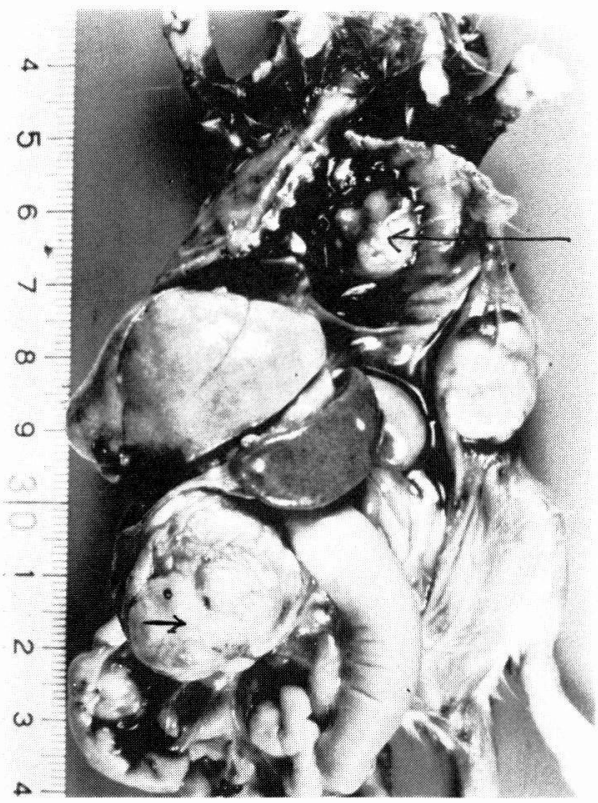

b

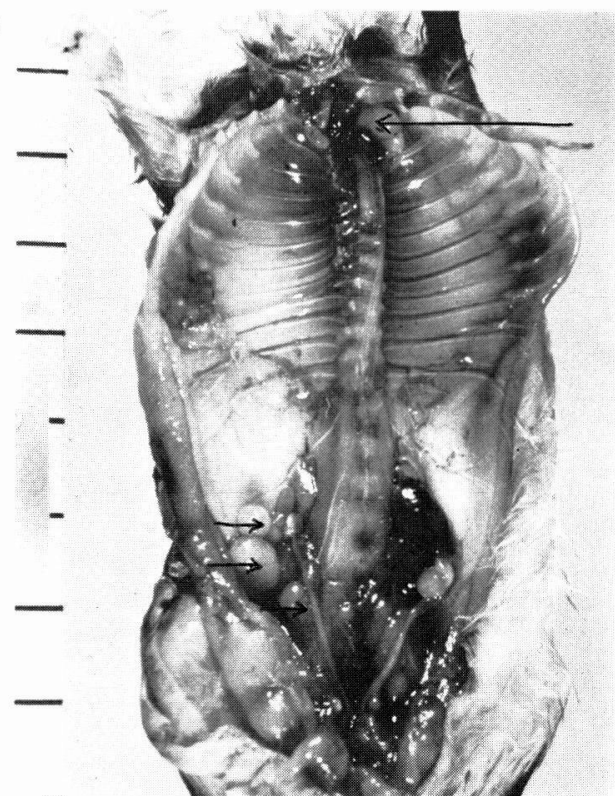

Fig. 2. Rats sacrificed at 6 weeks after transplantation.

a) The transplanted tumor (short arrow) had progressed beyond the peritoneal cavity. A lung metastasis (long arrow) was observed.

b) A Lymph node metastasis (short arrow) and Virchow's metastasis (long arrow) were seen. 


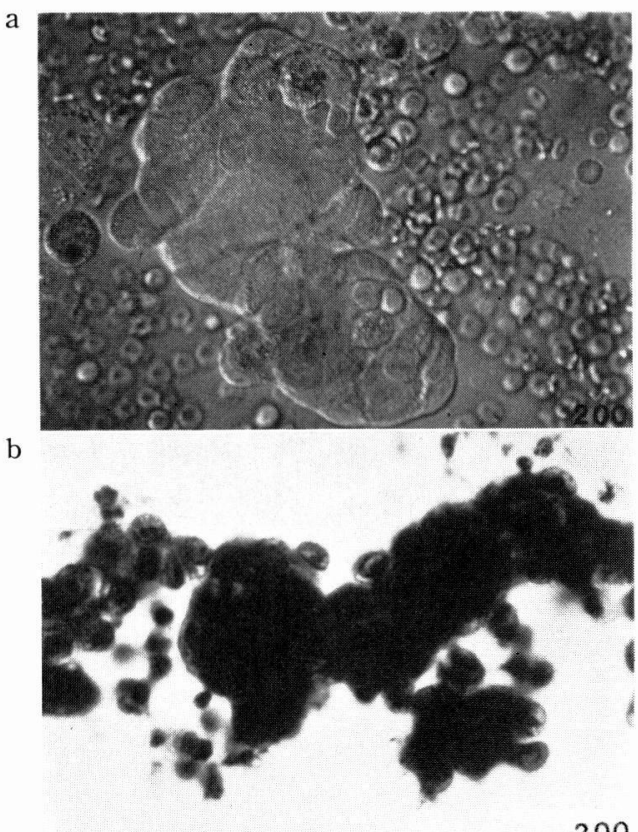

Fig. 3. A cluster of neoplastic cells, like a bunch of grapes, was observed with a) intravital stain and b) Papanicolaou's stain.

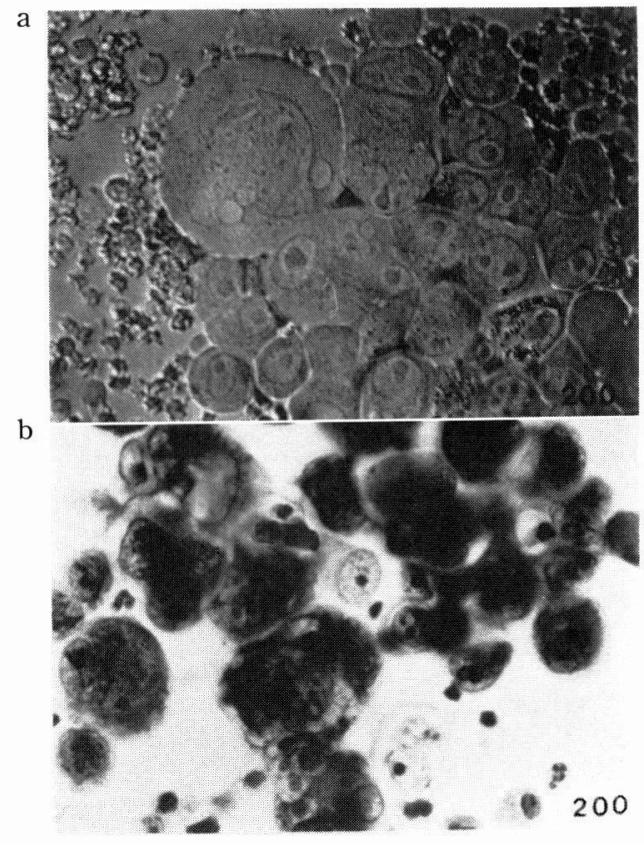

Fig. 5. The neoplastic cells lost their solidity and developed a flat arrangement, compared to Fig. 3 with a) intravital stain, and b) Papanicolaou's stain.

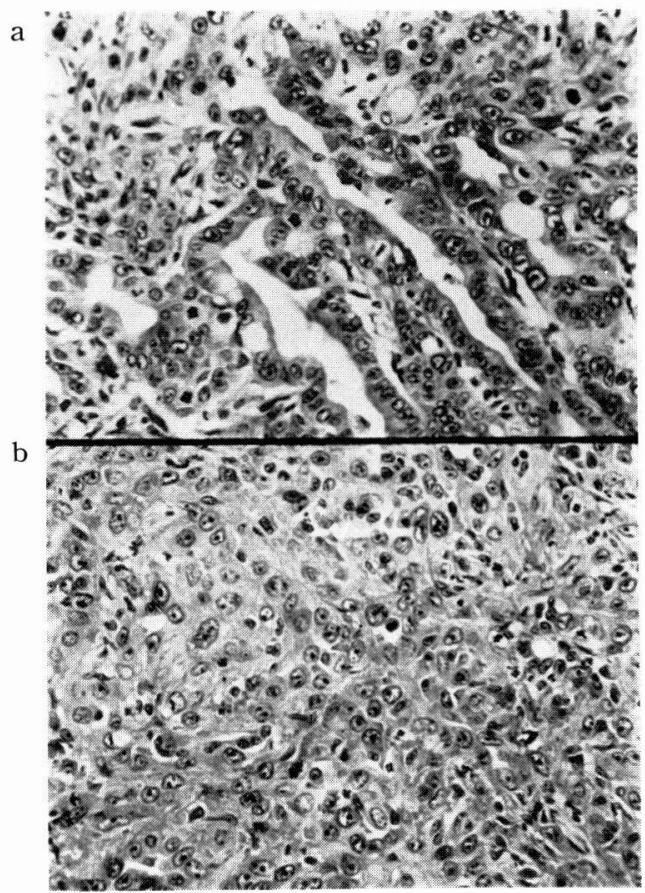

Fig. 4. The most common histology of DMBA induced ovarian cancer was a concomitant adenocarcinoma with glandular a) and solid b) structures. (HE, $\times 200)$

The histological specimens of the intraperitoneal transplants revealed adenocarcinomas with glandular and solid structures (Fig. 4)

The antitumor effects of cisplatin were indicated by a decrease in the ascites and in the histological and cytological damage.

Preliminary investigations revealed the following cytological antitumor effects.

1) Increased nucleated cells. The increase was due to lymphocytes and there was a decrease in cancer cells.

2) Decreased cell clusters which had lost their solidity and formed a flat arrangement. The number of isolated cells had increased (Fig. 5).

3) Larger sized cells.

4) More polynuclear cells with increased vacuoles in the cytoplasm (Fig. 6).

Histologically, the antitumor effects varied with the lesions in the abdominal cavity. Intra-abdominal administration 
a

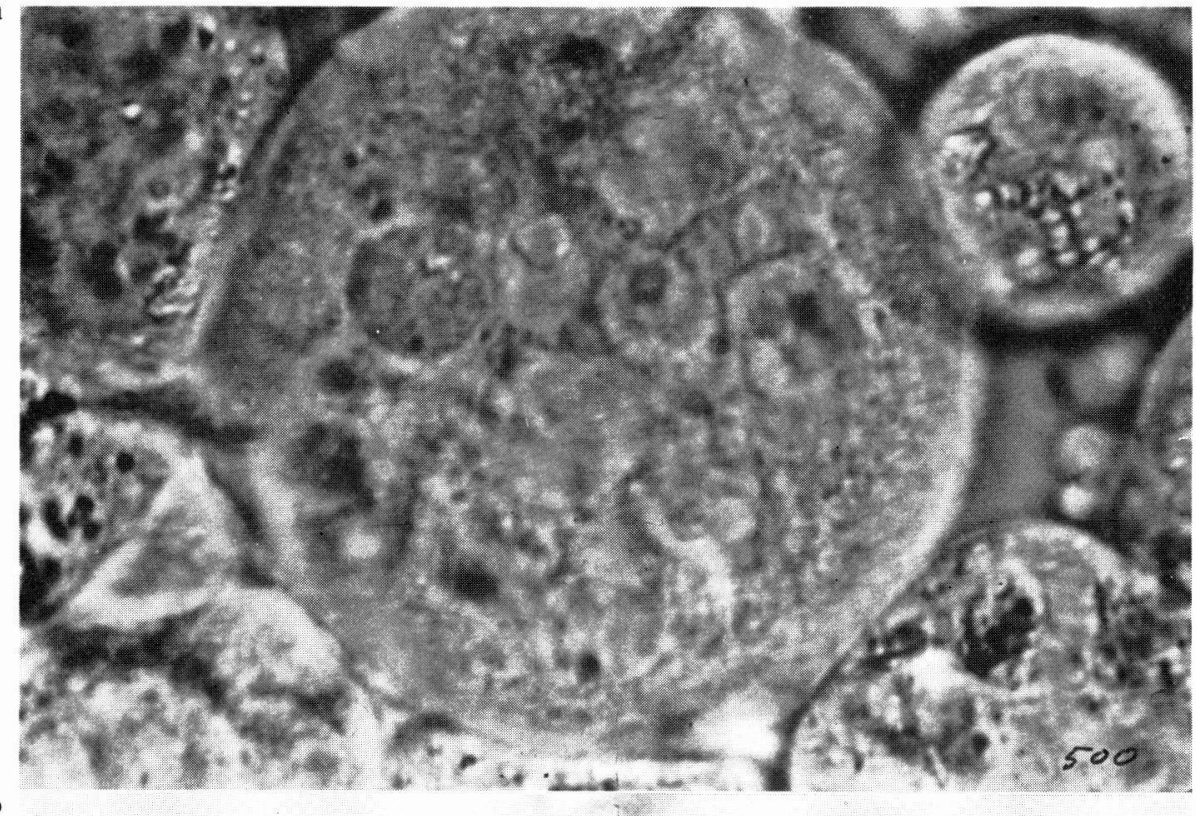

b

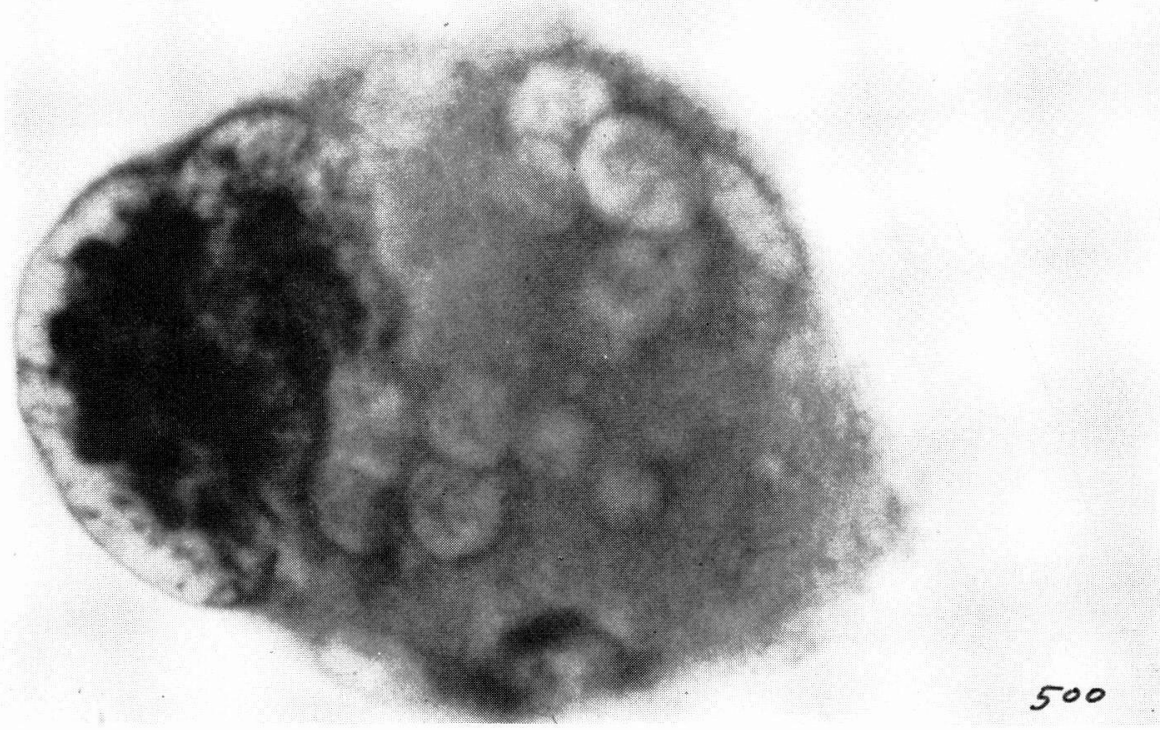

Fig. 6. The polynuclear cells (a) and multiple vacuoles in the cytoplasm (b) were remarkable with a) intravital stain, and b) Papanicolaou's stain. 


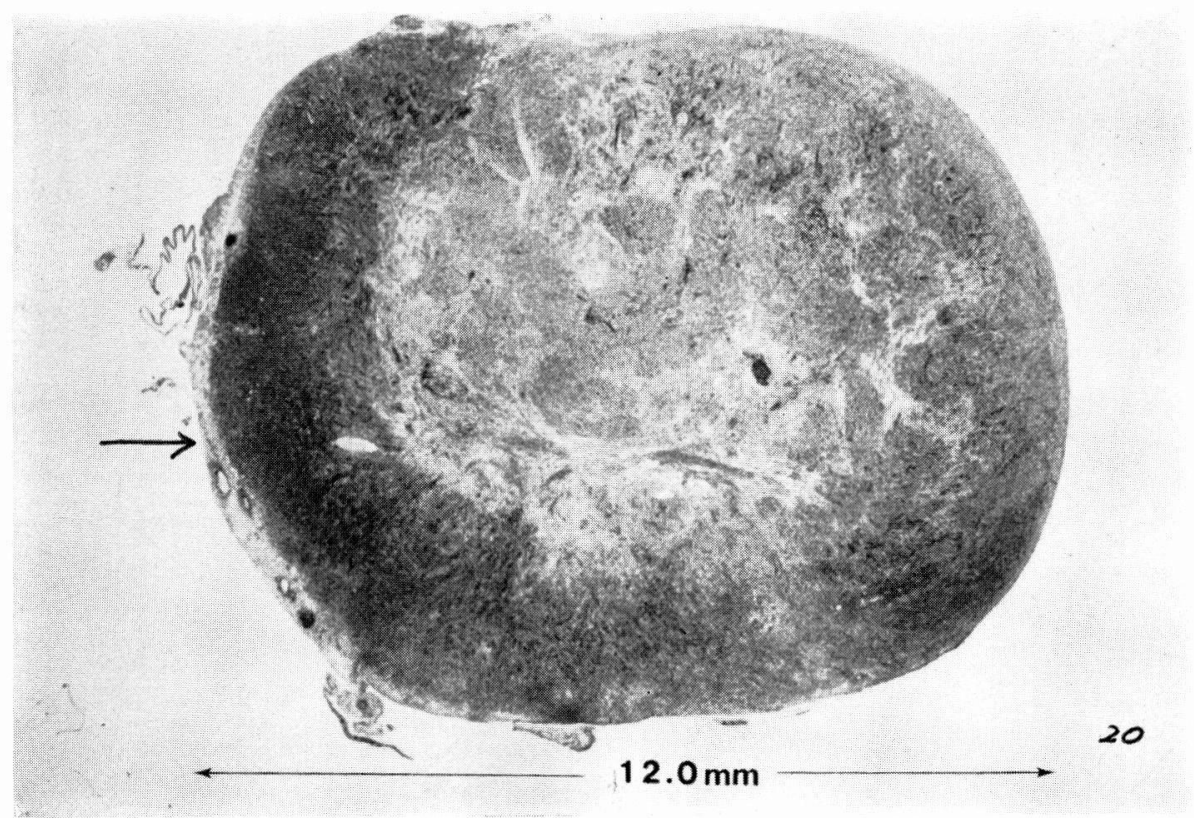

Fig. 7. The antitumor effects varied with the lesions in the abdominal tumor and were observed on the surface of the tumor (arrow). (HOPA $\times 20)$

produced a marked effect on the surface of the tumor (Fig. 7). The smaller the tumor, the more effective this administration was. However, lymph node metastases were scarcely affected.

\section{Discussion}

In recent years, treatment of advanced ovarian cancer has become a multi-disciplinary procedure with the introduction of debulking surgery and second look operations (SLO). In fact, the relationship between the diameter of the residual tumor and chemotherapy has been investigated, and the therapeutic effect can be assessed by SLO (Redman et al. 1986; Shepherd et al. 1986; Yakushiji, 1987). Moreover, a regimen of chemotherapy that is recognized to be effective needs to be formulated. The Japan Society For Cancer Therapy has proposed histological criteria for the evaluation of the effects of anti- tumor agents, but these are not necessarily satisfactory (Noda, 1988). Furthermore, there is a growing need for detailed assessment of antitumor effects at the cellular level in ascites and peritoneal washing fluid. For these reasons, appropriate animal models are required to achieve better clinical results. Although a number of experimentally induced ovarian tumors have been successfully transplanted, most of them were in the teratoma and granulosa cell tumor categories, both of which are comparatively rare in the human ovary.

A primary ovarian cancer has been produced in rats by direct intra-ovarian application of DMBA, and was subcultured by subcutaneous grafting into the backs of allogenic animals (Kato et al. 1974; Sugiyama et al. 1987)

In the present study, the tumor was allogenically transplanted, intraperitoneally. The tumor growing in the peritoneal cavity after transplantation was a 
concomitant adenocarcinoma identical to the primary lesion. It also spread by dissemination and lymph node metastasis, and was accompanied by malignant ascites, though the rats lacked a greater omentum. This method allowed for the production of a model for an advanced cancer corresponding to FIGO stage III-IV in large guantities with sufficient reproducibility within a short period of 4-6 weeks. The implant technique was sufficiently rapid to make large scale experiments possible. In addition, the original tumor was a primary allogenic ovarian adenocarcinoma with morphological similarities to human cancer. These observations highlight the reproducibility, resemblance and predictability of this model and its usefulness in experiments. Although an antitumor agent, cisplatin, was administered intraperitoneally to only a small number of animals; this preliminary evaluation was sufficient to demonstrate cytological as well as histological signs of cisplatin efficacy, suggesting that the model could be used for basic evaluation of drug treatments.

In fact, the intraperitoneal administration of cisplatin produced no uniform effect on the lesions in the abdominal cavity, but it can be expected to affect the outermost layer of the tumor where the cellular density is high. This indicates, as stated by Markman et al. (1984), that it is effective for minimal disease. While reports on cytological findings are scanty, findings were obtained that corresponded to morphological changes (Okawada et al. 1987).

Detailed studies in the future will allow these findings to impact on clinical practice.

Acknowledgments: This work was supported in part by a Grant-in Aid from the Ministry of Education, Science and Culture of Japan.

\section{References}

Annual report on the results of treatment in gynecological cancer. Volume 19, FIGO, 1985.

Kato, T., Yakushigi, M., Tsunawaki, A. and Ide, K. (1974). Studies of experimental ovarian tumors. Ovarian tumors developed in rats receiving chemical carcinogen 9,10-dimethyl1,2-benzanthracene. Kurume Med. J. 21, 1119.

Markman, M. (1984). Combination intraperitoneal chemotherapy with cisplatain, cytarabine and doxorubicin for refractory ovarian carcinoma and other malignancies principally confined to the peritoneal cavity. J. Clin. Oncol. 2, 1321-1326.

Nishida, T., Sugiyama, T., Katabuchi, H., Yakushiji, M. and KATO, T. (1986). Histologic origin of rat ovarian cancer induced by direct application of 7,12-dimethylbenz (a) anthrancene. Acta Obst Gynaec Jpn. 38, 570-574.

NoDA, K. (1988). Proposed histopathologic criteria of antitumor effect. Oncology and Chemotherapy, 4, 320-325.

Okawada, M., Kubo, R., Kumai, K. and Asai, F. (1987). Morphologic changes of cancerous ascitic cells induced by anticancer drug therapy - Five cases reports of ovarian and endometrial cancers - J. Jpn. Soc. Clin. Cytol. 26, 134-140.

Redman, J.R., Petrini, G.R. and Saigo, P.E. (1986). Prognostic factors in advanced ovarian carcinoma. J. Clin. Oncol., 4, 515-523.

Shepherd. J. H., Crowther, M.E. and Ward, B.G. (1986). Surgical procedures for ovarian cancer. Annals of the Royal college of Surgions of England. 68, 315-318.

Sugiyama, T. (1981). Cancer chemotherapy model using autochthonous ovarian cancer in rats. Kurume Med. J. 44, 731-747.

Sugiyama, T., Nishida, T., Nakanami, M., Ushijima, K., Shibue, K., Katabuchi. H., Yakushiji, M. and Kato, T. (1987). Characterization of transplant generations of primary rat adenocarcinoma and some chemotherapeutic observations. Acta Obst Gynaec Jpn, 39, 681-686.

Sugiyama, T., Nishida, T., Oda, T., Katabuchi, H., Kamura, N., Araki, T., Yakushiji, M. and Kato, T. (1985). Cancer chemotherapy model uing autochthonous ovarian cancer in rats. J. Jpn. Soc. Cancer Ther. 20, 1271-1278.

Yakushiji, M. (1987). Current role of chemotherapy in the management of ovarian cancer. Seminars in Surgical Oncology. 3, 45-54. 